\title{
Negative bases and automata
}

\author{
Christiane Frougny ${ }^{1}$ and Anna Chiara Lai ${ }^{2}$ \\ ${ }^{1}$ LIAFA, CNRS UMR 7089, Paris, France, and University Paris 8 \\ ${ }^{2}$ SBAI, Università La Sapienza, Rome, Italy \\ received $10^{\text {th }}$ December 2010, accepted $15^{\text {th }}$ March 2011.
}

\begin{abstract}
We study expansions in non-integer negative base $-\beta$ introduced by Ito and Sadahiro. Using countable automata associated with $(-\beta)$-expansions, we characterize the case where the $(-\beta)$-shift is a system of finite type. We prove that, if $\beta$ is a Pisot number, then the $(-\beta)$-shift is a sofic system. In that case, addition (and more generally normalization on any alphabet) is realizable by a finite transducer. We then give an on-line algorithm for the conversion from positive base $\beta$ to negative base $-\beta$. When $\beta$ is a Pisot number, the conversion can be realized by a finite on-line transducer.
\end{abstract}

Keywords: numeration system, sofic system, Pisot number, automaton, transducer

\section{Introduction}

Expansions in integer negative base $-b$, where $b \geqslant 2$, seem to have been introduced by Grünwald in [8], and rediscovered by several authors, see the historical comments given by Knuth [12]. The choice of a negative base $-b$ and of the alphabet $\{0, \ldots, b-1\}$ is interesting, because it provides a signless representation for every number (positive or negative). In this case it is easy to distinguish the sequences representing a positive integer from the ones representing a negative integer: denoting $\left(w_{\bullet}\right)_{-b}:=\sum_{i=0}^{k} w_{i}(-b)^{i}$ for any $w=w_{k} \cdots w_{0}$ in $\{0, \ldots, b-1\}^{*}$ with no leading 0 's, we have $\mathbb{N}=\left\{\left(w_{\bullet}\right)_{-b}|| w \mid\right.$ is odd $\}$. The classical monotonicity between the lexicographical ordering on words and the represented numerical values does not hold anymore in negative base, for instance $3=(111)_{-2}, 4=(100)_{-2}$ and $111>_{\text {lex }} 100$. Nevertheless it is possible to restore such a correspondence by introducing an appropriate ordering on words, in the sequel denoted by $\prec$ alt, and called the alternate order.

Representations in negative base also appear in some complex base number systems, for instance base $\beta=2 i$ since $\beta^{2}=-4$ (see [5] for a study of their properties from an automata theoretic point of view). Thus, beyond the interest in the problem in itself, the authors also wish the study of negative bases to be a useful preliminar step to better understanding the complex case.

Ito and Sadahiro recently introduced expansions in non-integer negative base $-\beta$ in [10]. They have given a characterization of admissible sequences, and shown that the $(-\beta)$-shift is sofic if and only if the $(-\beta)$-expansion of the number $-\frac{\beta}{\beta+1}$ is eventually periodic.

In this paper we pursue their work. The purpose of this contribution is to show that many properties of the positive base (integer or not) numeration systems extend to the negative base case, the main difference 1365-8050 (c) 2011 Discrete Mathematics and Theoretical Computer Science (DMTCS), Nancy, France 
being the sets of numbers that are representable in the two different cases. The results could seem not surprising, but this study put into light the important role played by the order on words: the lexicographic order for the positive bases, the alternate order for the negative bases.

Very recently there have been several contributions to the study of numbers having only positive powers of the base in their expansion, the so-called $(-\beta)$-integers, in [1], [16], and [23]. Dynamical properties of the $(-\beta)$-transformation are studied in [13].

We first establish some properties of the negative integer base $-b$, that are more or less folklore. This allows us to introduce the definitions of alternate order and of short-alternate order, that make possible to order numbers by their $(-\beta)$-expansions.

We then prove a general result which is not related to numeration systems but to the alternate order, and which is of interest in itself. We define a symbolic dynamical system associated with a given infinite word $s$ satisfying some properties with respect to the alternate order on infinite words. We design an infinite countable automaton recognizing it. We then are able to characterize the case when the symbolic dynamical system is sofic (resp. of finite type). Using this general construction we can prove that the $(-\beta)$-shift is a symbolic dynamical system of finite type if and only if the $(-\beta)$-expansion of $-\frac{\beta}{\beta+1}$ is purely periodic. We also show that the entropy of the $(-\beta)$-shift is equal to $\log \beta$.

We then focus on the case where $\beta$ is a Pisot number, that is to say, an algebraic integer greater than 1 such that the modulus of its Galois conjugates is less than 1. The natural integers and the Golden Mean are Pisot numbers. We extend all the results known to hold true in the Pisot case for $\beta$-expansions to the $(-\beta)$-expansions. In particular we prove that, if $\beta$ is a Pisot number, then every number from $\mathbb{Q}(\beta)$ has an eventually periodic $(-\beta)$-expansion, and thus that the $(-\beta)$-shift is a sofic system.

When $\beta$ is a Pisot number, it is known that addition in base $\beta$ - and more generally normalization in base $\beta$ on an arbitrary alphabet - is realizable by a finite transducer [4]. We show that this is still the case in base $-\beta$.

The conversion from positive integer base to negative integer base is realizable by a finite right sequential transducer. When $\beta$ is not an integer, we give an on-line algorithm for the conversion from base $\beta$ to base $-\beta$, where the result is not admissible in general. When $\beta$ is a Pisot number, the conversion can be realized by a finite on-line transducer.

A preliminary version of Sections 4 and 5 has been presented in [6].

\section{Definitions and preliminaries}

\subsection{Words and automata}

An alphabet is a totally ordered set. In this paper the alphabets are always finite. A finite sequence of elements of an alphabet $A$ is called a word, and the set of words over $A$ is the free monoid $A^{*}$. The empty word is denoted by $\varepsilon$. The set of infinite (resp. bi-infinite) words over $A$ is denoted by $A^{\mathbb{N}}$ (resp. $A^{\mathbb{Z}}$ ). Let $v$ be a word of $A^{*}$, denote by $v^{n}$ the concatenation of $v$ to itself $n$ times, and by $v^{\omega}$ the infinite concatenation $v v v \cdots$. A word of the form $u v^{\omega}$ is said to be eventually periodic. A (purely) periodic word is an eventually periodic word of the form $v^{\omega}$.

A finite word $v$ is a factor of a (finite, infinite or bi-infinite) word $x$ if there exists $u$ and $w$ such that $x=u v w$. When $u$ is the empty word, $v$ is a prefix of $x$. The prefix $v$ is strict if $v \neq x$. When $w$ is empty, $v$ is said to be a suffix of $x$.

We recall some definitions on automata, see [3] and [20] for instance. An automaton over $A, \mathcal{A}=$ 
$(Q, A, E, I, T)$, is a directed graph labelled by elements of $A$. The set of vertices, traditionally called states, is denoted by $Q, I \subset Q$ is the set of initial states, $T \subset Q$ is the set of terminal states and $E \subset Q \times A \times Q$ is the set of labelled edges. If $(p, a, q) \in E$, we write $p \stackrel{a}{\rightarrow} q$. The automaton is finite if $Q$ is finite. The automaton $\mathcal{A}$ is deterministic if $E$ is the graph of a (partial) function from $Q \times A$ into $Q$, and if there is a unique initial state. A subset $H$ of $A^{*}$ is said to be recognizable by a finite automaton, or regular, if there exists a finite automaton $\mathcal{A}$ such that $H$ is equal to the set of labels of paths starting in an initial state and ending in a terminal state.

Recall that two words $u$ and $v$ are said to be right congruent modulo $H$ if, for every $w, u w$ is in $H$ if and only if $v w$ is in $H$. It is well known that $H$ is recognizable by a finite automaton if and only if the congruence modulo $H$ has finite index.

Let $A$ and $A^{\prime}$ be two alphabets. A transducer is an automaton $\mathcal{T}=\left(Q, A^{*} \times A^{* *}, E, I, T\right)$ where the edges of $E$ are labelled by pairs in $A^{*} \times A^{* *}$. It is said to be finite if the set $Q$ of states and the set $E$ of edges are finite. If $(p,(u, v), q) \in E$, we write $p \stackrel{u \mid v}{\longrightarrow} q$. The input automaton (resp. output automaton) of such a transducer is obtained by taking the projection of edges on the first (resp. second) component. A transducer is said to be sequential if its input automaton is deterministic.

An on-line transducer is a particular kind of sequential transducer. An on-line transducer with delay $\delta, \mathcal{A}=\left(Q, A \times\left(A^{\prime} \cup \varepsilon\right), E,\left\{q_{0}\right\}\right)$, is a sequential automaton composed of a transient part and of a synchronous part, see [17]. The set of states is equal to $Q=Q_{t} \cup Q_{s}$, where $Q_{t}$ is the set of transient states and $Q_{s}$ is the set of synchronous states. In the transient part, every path of length $\delta$ starting in the initial state $q_{0}$ is of the form

$$
q_{0} \stackrel{x_{1} \mid \varepsilon}{\longrightarrow} q_{1} \stackrel{x_{2} \mid \varepsilon}{\longrightarrow} \cdots \stackrel{x_{\delta} \mid \varepsilon}{\longrightarrow} q_{\delta}
$$

where $q_{0}, \ldots, q_{\delta-1}$ are in $Q_{t}, x_{j}$ in $A$, for $1 \leqslant j \leqslant \delta$, and the only edge arriving in a state of $Q_{t}$ is as above. In the synchronous part, edges are labelled by elements of $A \times A^{\prime}$. This means that the transducer starts reading words of length $\leqslant \delta$ and outputting nothing, and after that delay, outputs serially one digit for each input digit. If the set of states $Q$ and the set of edges $E$ are finite, the on-line automaton is said to be finite.

The same notions can be defined for automata and transducer processing words from right to left : they are called right automata or transducers.

\subsection{Symbolic dynamics}

Let us recall some definitions on symbolic dynamical systems or subshifts (see [15, Chapter 1] or [14]). The set $A^{\mathbb{Z}}$ is endowed with the lexicographic order, denoted $<_{l e x}$, the product topology, and the shift $\sigma$, defined by $\sigma\left(\left(x_{i}\right)_{i \in \mathbb{Z}}\right)=\left(x_{i+1}\right)_{i \in \mathbb{Z}}$. A set $S \subseteq A^{\mathbb{Z}}$ is a symbolic dynamical system, or subshift, if it is shift-invariant and closed for the product topology on $A^{\mathbb{Z}}$. A bi-infinite word $z$ avoids a set of word $X \subset A^{*}$ if no factor of $z$ is in $X$. The set of all words which avoid $X$ is denoted $S_{X}$. A set $S \subseteq A^{\mathbb{Z}}$ is a subshift if and only if $S$ is of the form $S_{X}$ for some $X$.

The same notion can be defined for a one-sided subshift of $A^{\mathbb{N}}$.

Let $F(S)$ be the set of factors of elements of $S$, let $I(S)=A^{+} \backslash F(S)$ be the set of words avoided by $S$, and let $X(S)$ be the set of elements of $I(S)$ which have no proper factor in $I(S)$. The subshift $S$ is sofic if and only if $F(S)$ is recognizable by a finite automaton, or equivalently if $X(S)$ is recognizable by a finite automaton. The subshift $S$ is of finite type if $S=S_{X}$ for some finite set $X$, or equivalently if $X(S)$ is finite. We will say that the subshift $S$ is recognizable by an automaton $\mathcal{A}$ (finite or infinite) when the set $F(S)$ is recognizable by $\mathcal{A}$. 
The topological entropy of a subshift $S$ is

$$
h(S)=\lim _{n \rightarrow \infty} \frac{1}{n} \log \left(B_{n}(S)\right)
$$

where $B_{n}(S)$ is the number of elements of $F(S)$ of length $n$. When $S$ is sofic, the entropy of $S$ is equal to the logarithm of the spectral radius of the adjacency matrix of the finite automaton recognizing $F(S)$.

\subsection{Numeration systems}

The reader is referred to [15, Chapter 7] and to [7] for a detailed presentation of these topics. Representations of real numbers in a non-integer base $\beta>1$ were introduced by Rényi [19] under the name of $\beta$ expansions. Let $x$ be a real number in the interval $[0,1]$. A representation in base $\beta$ (or a $\beta$-representation) of $x$ is an infinite word $\left(x_{i}\right)_{i \geqslant 1}$ such that

$$
x=\sum_{i \geqslant 1} x_{i} \beta^{-i} .
$$

Let $\mathbf{x}=\left(x_{i}\right)_{i \geqslant 1}$. The numerical value in base $\beta$ is the function $\pi_{\beta}$ defined by $\pi_{\beta}(\mathbf{x})=\sum_{i=1}^{\infty} x_{i} \beta^{-i}$.

A particular $\beta$-representation - called the $\beta$-expansion — of a real number $x$ in $[0,1]$ can be computed by the "greedy algorithm" : denote by $\lfloor y\rfloor,\lceil y\rceil$ and $\{y\}$ the lower integer part, the upper integer part and the fractional part of a number $y$. Set $r_{0}=x$ and let for $i \geqslant 1, x_{i}=\left\lfloor\beta r_{i-1}\right\rfloor, r_{i}=\left\{\beta r_{i-1}\right\}$. Then $x=\sum_{i \geqslant 1} x_{i} \beta^{-i}$. The $\beta$-expansion of $x$ will be denoted by $\mathrm{d}_{\beta}(x)=\left(x_{i}\right)_{i \geqslant 1}$.

The digits $x_{i}$ are elements of the canonical alphabet $A_{\beta}=\{0, \ldots,\lceil\beta\rceil-1\}$, excepted when $\beta$ is an integer and $x=1$, in which case $\mathrm{d}_{\beta}(1)=\beta 000 \ldots$.

If $x>1$, there exists some $k \geqslant 1$ such that $x / \beta^{k}$ belongs to $[0,1)$. If $\mathrm{d}_{\beta}\left(x / \beta^{k}\right)=\left(y_{i}\right)_{i \geqslant 1}$ then, by shifting, the $\beta$-expansion of $x$ is $\langle x\rangle_{\beta}=y_{1} \cdots y_{k} . y_{k+1} y_{k+2} \cdots$.

An equivalent definition is obtained by using the $\beta$-transformation of the unit interval which is the mapping

$$
T_{\beta}: x \mapsto \beta x-\lfloor\beta x\rfloor .
$$

Then $\mathrm{d}_{\beta}(x)=\left(x_{i}\right)_{i \geqslant 1}$ if and only if $x_{i}=\left\lfloor\beta T_{\beta}^{i-1}(x)\right\rfloor$.

If a representation ends in infinitely many zeros, like $v 0^{\omega}$, the ending zeros are omitted and the representation is said to be finite.

In the case where the $\beta$-expansion of 1 is finite, there is a special representation playing an important role. Let $\mathrm{d}_{\beta}(1)=\left(t_{i}\right)_{i \geqslant 1}$ and set $\mathrm{d}_{\beta}^{*}(1)=\mathrm{d}_{\beta}(1)$ if $\mathrm{d}_{\beta}(1)$ is infinite and $\mathrm{d}_{\beta}^{*}(1)=\left(t_{1} \cdots t_{m-1}\left(t_{m}-1\right)\right)^{\omega}$ if $\mathrm{d}_{\beta}(1)=t_{1} \cdots t_{m-1} t_{m}$ is finite.

A word $\left(x_{i}\right)_{i \geqslant 1}$ is said to be $\beta$-admissible if there exists a real number $x \in[0,1)$ such that $\mathrm{d}_{\beta}(x)=$ $\left(x_{i}\right)_{i \geqslant 1}$. Denote by $D_{\beta}$ the set of $\beta$-expansions of numbers of $[0,1)$. It is a shift-invariant subset of $A_{\beta}^{\mathbb{N}}$. The $\beta$-shift $S_{\beta}$ is the closure of $D_{\beta}$ and it is a subshift of $A_{\beta}^{\mathbb{Z}}$. When $\beta$ is an integer, $S_{\beta}$ is the full $\beta$-shift $A_{\beta}^{\mathbb{Z}}$.

Theorem 2.1 (Parry[18]) Let $\beta>1$ be a real number. A word $\left(w_{i}\right)_{i \geqslant 1}$ belongs to $D_{\beta}$ if and only if for all $n \geqslant 1$

$$
w_{n} w_{n+1} \cdots<_{l e x} \mathrm{~d}_{\beta}^{*}(1) .
$$

A word $\left(w_{i}\right)_{i \in \mathbb{Z}}$ belongs to $S_{\beta}$ if and only if for all $n$

$$
w_{n} w_{n+1} \cdots \leqslant \leqslant_{l e x} \mathrm{~d}_{\beta}^{*}(1) .
$$


The following results are well-known (see [15, Chapt. 7]).

Theorem 2.2 1. The $\beta$-shift is sofic if and only if $\mathrm{d}_{\beta}(1)$ is eventually periodic.

2. The $\beta$-shift is of finite type if and only if $\mathrm{d}_{\beta}(1)$ is finite.

It is known that the entropy of the $\beta$-shift is equal to $\log \beta$, [11].

If $\beta$ is a Pisot number, then every element of $\mathbb{Q}(\beta) \cap[0,1]$ has an eventually periodic $\beta$-expansion, and the $\beta$-shift $S_{\beta}$ is a sofic system $[2,21]$.

Let $C$ be an arbitrary finite alphabet of integer digits. The normalization function in base $\beta$ on $C$

$$
\nu_{\beta, C}: C^{\mathbb{N}} \rightarrow \mathcal{A}_{\beta}^{\mathbb{N}}
$$

is the partial function which maps an infinite word $\mathbf{y}=\left(y_{i}\right)_{i \geqslant 1}$ over $C$, such that $0 \leqslant y=\sum_{i \geqslant 1} y_{i} \beta^{-i} \leqslant$ 1 , onto the $\beta$-expansion of $y$. It is known [4] that, when $\beta$ is a Pisot number, normalization is computable by a finite transducer on any alphabet $C$. Note that addition is a particular case of normalization, with $C=\{0, \ldots, 2(\lceil\beta\rceil-1)\}$.

\section{Negative integer base}

Let $b>1$ be an integer. It is well known, see Knuth [12] for instance, that every integer (positive or negative) has a unique $(-b)$-expansion with digits in $A_{b}=\{0,1, \ldots, b-1\}$. Every real number (positive or negative) has a $(-b)$-representation, not necessarily unique, since

$$
\pi_{-b}\left(1((b-1) 0)^{\omega}\right)=\pi_{-b}\left(0(0(b-1))^{\omega}\right)=-\frac{1}{b(b+1)}
$$

for instance.

We recall some well-known facts.

Proposition 3.1 The set of $(-b)$-expansions of the positive integers is $\left\{u \in\{0,1, \ldots, b-1\}^{*} \mid u\right.$ does not begin with 0 and $|u|$ is odd $\}$. The set of $(-b)$-expansions of the negative integers is $\{u \in\{0,1, \ldots, b-$ $1\}^{*} \mid u$ does not begin with 0 and $|u|$ is even $\}$.

Let $A$ be a finite alphabet totally ordered, and let $\min A$ be its smallest element.

Definition 3.2 The alternate order $\prec$ alt on infinite words or finite words with same length over $A$ is defined by:

$$
u_{1} u_{2} u_{3} \ldots \prec_{\text {alt }} v_{1} v_{2} v_{3} \ldots
$$

if and only if there exists $k \geqslant 1$ such that

$$
u_{i}=v_{i} \text { for } 1 \leqslant i<k \text { and }(-1)^{k}\left(u_{k}-v_{k}\right)<0 .
$$

This order was implicitely defined in [8].

Definition 3.3 On the set of finite words, we define the short-alternate order, denoted $\prec_{s a}$, by: if $u=$ $u_{1} \cdots u_{\ell}$ and $v=v_{1} \cdots v_{m}$ are in $A^{*}$, then $u \prec_{s a} v$ if and only if

- $\ell$ and $m$ are odd, and $\ell<m$, or $\ell=m$ and $(\min A) u \prec_{\text {alt }}(\min A) v$ 
- $\ell$ and $m$ are even, and $\ell>m$, or $\ell=m$ and $u \prec_{\text {alt }} v$

- $\ell<m$ and $(\min A)^{m-\ell} u \prec_{s a} v$

- $\ell>m$ and $u \prec_{s a}(\min A)^{\ell-m} v$.

The short-alt order is analogous to the short-lex or radix order relatively to the lexicographical order. Denote $\langle x\rangle_{-b}$ the $(-b)$-expansion of $x$. We have the following result.

Proposition 3.4 If $x$ and $y$ are integers, $x<y$ if and only if $\langle x\rangle_{-b} \prec_{s a}\langle y\rangle_{-b}$.

Example 3.5 In base $-2,\langle 3\rangle_{-2}=111,\langle 4\rangle_{-2}=100,\langle 6\rangle_{-2}=11010$, and $111 \prec_{\text {sa }} 100 \prec_{\text {sa }} 11010$.

Proposition 3.6 The function that maps the b-expansion of a positive integer to its $(-b)$-expansion can be realized by a finite right sequential transducer.

Proof: In Fig. $1,0 \leqslant c \leqslant b-1,1 \leqslant d \leqslant b-1$, and $0 \leqslant e \leqslant b-2$. The processing is done from right to left by 2-letter blocks. A finite word of the form $x_{2 k-1} \cdots x_{0}$ which is the $b$-expansion of $x$ prefixed by enough 0 's is transformed by the transducer into a finite word $y_{2 k-1} \cdots y_{0}$ which is the $(-b)$-expansion of $x$, maybe prefixed by 0 's. It is straightforward to transform this transducer into a finite right sequential transducer.

$$
\begin{gathered}
(b-d-1)(b-c-1) \\
0(b-1) \mid(b-1) 0
\end{gathered}
$$

Fig. 1: Finite right sequential transducer realizing conversion from base $b$ to base $-b$

Example 3.7 Conversion from base 2 to base -2 .

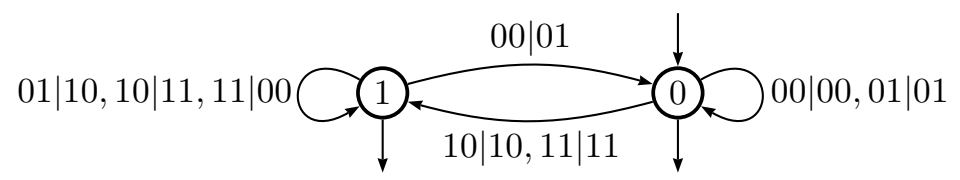

Fig. 2: Finite right sequential transducer realizing conversion from base 2 to base -2

\section{Symbolic dynamical systems and the alternate order}

We have seen in the previous section that the alternate order is the tool to compare numbers written in a negative base. In this section we give general results on symbolic dynamical systems defined by the alternate order. This is analogous to the symbolic dynamical systems defined by the lexicographical order, see [7]. Let $A$ be a totally ordered finite alphabet. 
Definition 4.1 $A$ word $s=s_{1} s_{2} \cdots$ in $A^{\mathbb{N}}$ is said to be an alternately shift minimal word (asmin-word for short) if $s_{1}=\max A$ and $s$ is smaller than, or equal to, any of its shifted images in the alternate order: for each $n \geqslant 1, s \preceq$ alt $s_{n} s_{n+1} \cdots$.

Let

$$
S(s)=\left\{w=\left(w_{i}\right)_{i \in \mathbb{Z}} \in A^{\mathbb{Z}} \mid \forall n, s \preceq_{a l t} w_{n} w_{n+1} \cdots\right\}
$$

be the subshift defined by the alternately shift minimal word $s$. We construct a countable infinite automaton $\mathcal{A}_{S(s)}$ as follows (see Fig. 3, where $[a, b]$ denotes the set $\{a, a+1, \ldots, b\}$ if $a \leqslant b$, $\varepsilon$ otherwise. It is assumed in Fig. 3 that $s_{1}>s_{j}$ for $j \geqslant 2$.) The set of states is $\mathbb{N}$, the initial state is 0 and every state is terminal. For each state $i \geqslant 0$, there is an edge $i \stackrel{s_{i+1}}{\longrightarrow} i+1$. If $i$ is even, then for each $a$ such that $0 \leqslant a \leqslant s_{i+1}-1$, there is an edge $i \stackrel{a}{\longrightarrow} j$, where $j$ is such that $s_{1} \cdots s_{j}$ is the suffix of maximal length of $s_{1} \cdots s_{i} a$. If $i$ is odd, then for each $b$ such that $s_{i+1}+1 \leqslant b \leqslant s_{1}-1$, there is an edge $i \stackrel{b}{\longrightarrow} j$ where $j$ is maximal such that $s_{1} \cdots s_{j}$ is a suffix of $s_{1} \cdots s_{i} b$; and if $s_{i+1}<s_{1}$ there is one edge $i \stackrel{s_{1}}{\longrightarrow} 1$. By contruction, the deterministic automaton $\mathcal{A}_{S(s)}$ recognizes exactly the words $w$ such that every suffix $y$ of $w$ is $\succeq_{\text {alt }} s_{1} \cdots s_{|y|}$ and the result below follows.

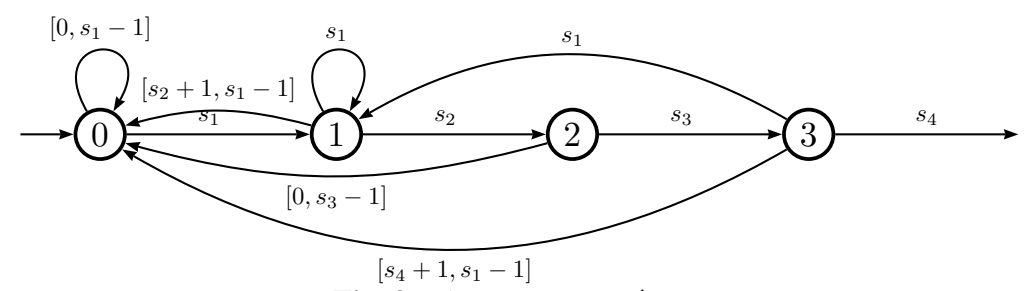

Fig. 3: The automaton $\mathcal{A}_{S(s)}$

Proposition 4.2 The subshift $S(s)=\left\{w=\left(w_{i}\right)_{i \in \mathbb{Z}} \in A^{\mathbb{Z}} \mid \forall n, s \preceq_{\text {alt }} w_{n} w_{n+1} \cdots\right\}$ is recognizable by the countable infinite automaton $\mathcal{A}_{S(s)}$.

Proposition 4.3 The subshift $S(s)=\left\{w=\left(w_{i}\right)_{i \in \mathbb{Z}} \in A^{\mathbb{Z}} \mid \forall n, s \preceq\right.$ alt $\left.w_{n} w_{n+1} \cdots\right\}$ is sofic if and only if $s$ is eventually periodic.

Proof: The subshift $S(s)$ is sofic if and only if the set of its finite factors $F(S(s))$ is recognizable by a finite automaton. Given a word $u$ of $A^{*}$, denote by $[u]$ the right class of $u$ modulo $F(S(s))$. Then in the automaton $\mathcal{A}_{S(s)}$, for each state $i \geqslant 1, i=\left[s_{1} \cdots s_{i}\right]$, and $0=[\varepsilon]$. Suppose that $s$ is eventually periodic, $s=s_{1} \cdots s_{m}\left(s_{m+1} \cdots s_{m+p}\right)^{\omega}$, with $m$ and $p$ minimal. Thus, for each $k \geqslant 0$ and each $0 \leqslant i \leqslant p-1$, $s_{m+p k+i}=s_{m+i}$.

Case 1: $p$ is even. Then for every $k \geqslant 0$ and $0 \leqslant i \leqslant p-1,\left[s_{1} \cdots s_{m+i}\right]=\left[s_{1} \cdots s_{m+p k+i}\right]$, thus the states $m+i$ and $m+p k+i$ can be merged. Then the set of states of $\mathcal{A}_{S(s)}$ is $\{0,1, \ldots, m+p-1\}$.

Case 2: $p$ is odd. Then $m+i=\left[s_{1} \cdots s_{m+i}\right]=\left[s_{1} \cdots s_{m+2 p k+i}\right]$ for every $k \geqslant 0$ and $0 \leqslant i \leqslant 2 p-1$, thus the states $m+i$ and $m+2 p k+i$ can be merged, and the set of states of $\mathcal{A}_{S(s)}$ is $\{0,1, \ldots, m+2 p-1\}$. Conversely, suppose that $s$ is not eventually periodic. Then there exists an infinite sequence of indices $i_{1}<i_{2}<\cdots$ such that the sequences $s_{i_{k}} s_{i_{k}+1} \cdots$ are all different for all $k \geqslant 1$. Take any pair $\left(i_{j}, i_{\ell}\right)$, $j, \ell \geqslant 1$. If $i_{j}$ and $i_{\ell}$ do not have the same parity, then $s_{1} \cdots s_{i_{j}}$ and $s_{1} \cdots s_{i_{\ell}}$ are not right congruent 
modulo $F(S(s))$. If $i_{j}$ and $i_{\ell}$ have the same parity, there exists $q \geqslant 0$ such that $s_{i_{j}} \cdots s_{i_{j}+q-1}=$ $s_{i_{\ell}} \cdots s_{i_{\ell}+q-1}=v$ and, for instance, $(-1)^{i_{j}+q}\left(s_{i_{j}+q}-s_{i_{\ell}+q}\right)>0$ (with the convention that, if $q=0$ then $v=\varepsilon)$. Then $s_{1} \cdots s_{i_{j}-1} v s_{i_{j}+q}$ and $s_{1} \cdots s_{i_{\ell}-1} v s_{i_{\ell}+q}$ both belong to $F(S(s))$, but $s_{1} \cdots s_{i_{j}-1} v s_{i_{\ell}+q}$ does not belong to $F(S(s))$. Hence $s_{1} \cdots s_{i_{j}}$ and $s_{1} \cdots s_{i_{\ell}}$ are not right congruent modulo $F(S(s))$, so the number of right congruence classes is infinite and $F(S(s))$ is thus not recognizable by a finite automaton.

Proposition 4.4 The subshift $S(s)=\left\{w=\left(w_{i}\right)_{i \in \mathbb{Z}} \in A^{\mathbb{Z}} \mid \forall n, s \preceq_{\text {alt }} w_{n} w_{n+1} \cdots\right\}$ is a subshift of finite type if and only if $s$ is purely periodic.

Proof: Suppose that $s=\left(s_{1} \cdots s_{p}\right)^{\omega}$. Consider the finite set $X=\left\{s_{1} \cdots s_{n-1} b \mid b \in A,(-1)^{n}(b-\right.$ $\left.\left.s_{n}\right)<0,1 \leqslant n \leqslant p\right\}$. We show that $S(s)=S(s)_{X}$. If $w$ is in $S(s)$, then $w$ avoids $X$, and conversely. Now, suppose that $S(s)$ is of finite type. It is thus sofic, and by Proposition $4.3 s$ is eventually periodic. If it is not purely periodic, then $s=s_{1} \cdots s_{m}\left(s_{m+1} \cdots s_{m+p}\right)^{\omega}$, with $m$ and $p$ minimal, and $s_{1} \cdots s_{m} \neq \varepsilon$. Let $I=\left\{s_{1} \cdots s_{n-1} b \mid b \in A,(-1)^{n}\left(b-s_{n}\right)<0,1 \leqslant n \leqslant m\right\} \cup$ $\left\{s_{1} \cdots s_{m}\left(s_{m+1} \cdots s_{m+p}\right)^{2 k} s_{m+1} \cdots s_{m+n-1} b \mid b \in A, k \geqslant 0,(-1)^{m+2 k p+n}\left(b-s_{m+n}\right)<0,1 \leqslant\right.$ $n \leqslant 2 p\}$. Then $I \subset A^{+} \backslash F(S(s))$. First, suppose there exists $1 \leqslant j \leqslant p$ such that $(-1)^{j}\left(s_{j}-s_{m+j}\right)<0$ and $s_{1} \cdots s_{j-1}=s_{m+1} \cdots s_{m+j-1}$. For $k \geqslant 0$ fixed, let $w^{(2 k)}=s_{1} \cdots s_{m}\left(s_{m+1} \cdots s_{m+p}\right)^{2 k} s_{1} \cdots s_{j} \in$ I. We have $s_{1} \cdots s_{m}\left(s_{m+1} \cdots s_{m+p}\right)^{2 k} s_{m+1} \cdots s_{m+j-1} \in F(S(s))$. On the other hand, for $n \geqslant 2$, $s_{n} \cdots s_{m}\left(s_{m+1} \cdots s_{m+p}\right)^{2 k}$ is greater in the alternate order than the prefix of $s$ of same length, thus $s_{n} \cdots s_{m}\left(s_{m+1} \cdots s_{m+p}\right)^{2 k} s_{1} \cdots s_{j}$ belongs to $F(S(s))$. Hence any strict factor of $w^{(2 k)}$ is in $F(S(s))$. Therefore for any $k \geqslant 0, w^{(2 k)} \in X(S(s))$, and $X(S(s))$ is thus infinite: $S(s)$ is not of finite type. Now, if such a $j$ does not exist, then for every $1 \leqslant j \leqslant p, s_{j}=s_{m+j}$, and $s=\left(s_{1} \cdots s_{m}\right)^{\omega}$ is purely periodic.

Remark 4.5 Let $s^{\prime}=s_{1}^{\prime} s_{2}^{\prime} \cdots$ be a word in $A^{\mathbb{N}}$ such that $s_{1}^{\prime}=\min$ A and, for each $n \geqslant 1, s_{n}^{\prime} s_{n+1}^{\prime} \cdots \preceq$ alt $s^{\prime}$. Such a word is said to be an alternately shift maximal word. Let $S^{\prime}\left(s^{\prime}\right)=\left\{w=\left(w_{i}\right)_{i \in \mathbb{Z}} \in A^{\overline{\mathbb{Z}}} \mid\right.$ $\forall n, w_{n} w_{n+1} \cdots \preceq$ alt $\left.s^{\prime}\right\}$. The statements in Propositions 4.2, 4.3 and 4.4 are also valid for the subshift $S^{\prime}\left(s^{\prime}\right)$ (with the automaton $\mathcal{A}_{S^{\prime}\left(s^{\prime}\right)}$ constructed accordingly).

\section{Negative real base}

\subsection{The $(-\beta)$-shift}

Ito and Sadahiro introduced in [10] a greedy algorithm to represent any real number in real base $-\beta$, $\beta>1$, and with digits in $A_{-\beta}=\{0,1, \ldots,\lfloor\beta\rfloor\}$. Remark that, when $\beta$ is not an integer, $A_{-\beta}=A_{\beta}$.

A transformation on $I_{-\beta}=\left[-\frac{\beta}{\beta+1}, \frac{1}{\beta+1}\right)$ is defined as follows:

$$
T_{-\beta}(x)=-\beta x-\left\lfloor-\beta x+\frac{\beta}{\beta+1}\right\rfloor .
$$

For every real number $x \in I_{-\beta}$ we will denote the $(-\beta)$-expansion of $x$ by $\mathrm{d}_{-\beta}(x)$. It is defined by $\mathrm{d}_{-\beta}(x)=\left(x_{i}\right)_{i \geqslant 1}$ if and only if $x_{i}=\left\lfloor-\beta T_{-\beta}^{i-1}(x)+\frac{\beta}{\beta+1}\right\rfloor$, and $x=\sum_{i \geqslant 1} x_{i}(-\beta)^{-i}$. When this last equality holds, we may also write:

$$
x=\left(\cdot x_{1} x_{2} \cdots\right)_{-\beta} .
$$


We show that the alternate order $\prec_{\text {alt }}$ on $(-\beta)$-expansions gives the numerical order.

Proposition 5.1 Let $x$ and $y$ be in $I_{-\beta}$. Then

$$
x<y \Longleftrightarrow \mathrm{d}_{-\beta}(x) \prec \text { alt } \mathrm{d}_{-\beta}(y) .
$$

Proof: Suppose that $\mathrm{d}_{-\beta}(x) \prec_{\text {alt }} \mathrm{d}_{-\beta}(y)$. Then there exists $k \geqslant 1$ such that $x_{i}=y_{i}$ for $1 \leqslant i<k$ and $(-1)^{k}\left(x_{k}-y_{k}\right)<0$. Suppose that $k$ is even, $k=2 q$. Then $x_{2 q} \leqslant y_{2 q}-1$. Thus $x-y \leqslant-\beta^{-2 q}+$ $\sum_{i \geqslant 2 q+1} x_{i}(-\beta)^{-i}-\sum_{i \geqslant 2 q+1} y_{i}(-\beta)^{-i}<0$, since $\sum_{i \geqslant 1} x_{2 q+i}(-\beta)^{-i}$ and $\sum_{i \geqslant 1} y_{2 q+i}(-\beta)^{-i}$ are in $I_{-\beta}$. The case $k=2 q+1$ is similar. The converse is immediate.

Definition $5.2 A$ word $\left(x_{i}\right)_{i \geqslant 1}$ is said to be $(-\beta)$-admissible if there exists a real number $x \in I_{-\beta}$ such that $\mathrm{d}_{-\beta}(x)=\left(x_{i}\right)_{i \geqslant 1}$. The $(-\beta)$-shift $S_{-\beta}$ is the closure of the set of $(-\beta)$-admissible words, and it is a subshift of $A_{\beta}^{\mathbb{Z}}$.

Define the sequence $\mathrm{d}_{-\beta}^{*}\left(\frac{1}{\beta+1}\right)$ as follows:

- if $\mathrm{d}_{-\beta}\left(-\frac{\beta}{\beta+1}\right)=d_{1} d_{2} \ldots$ is not a periodic sequence with odd period,

$$
\mathrm{d}_{-\beta}^{*}\left(\frac{1}{\beta+1}\right)=\mathrm{d}_{-\beta}\left(\frac{1}{\beta+1}\right)=0 d_{1} d_{2} \ldots
$$

- otherwise if $\mathrm{d}_{-\beta}\left(-\frac{\beta}{\beta+1}\right)=\left(d_{1} \cdots d_{2 p+1}\right)^{\omega}$,

$$
\mathrm{d}_{-\beta}^{*}\left(\frac{1}{\beta+1}\right)=\left(0 d_{1} \cdots d_{2 p}\left(d_{2 p+1}-1\right)\right)^{\omega} .
$$

Theorem 5.3 (Ito-Sadahiro [10]) $A$ word $\left(w_{i}\right)_{i \geqslant 1}$ is $(-\beta)$-admissible if and only if for each $n \geqslant 1$

$$
\mathrm{d}_{-\beta}\left(-\frac{\beta}{\beta+1}\right) \preceq \text { alt } w_{n} w_{n+1} \cdots \prec_{\text {alt }} \mathrm{d}_{-\beta}^{*}\left(\frac{1}{\beta+1}\right) .
$$

$A$ word $\left(w_{i}\right)_{i \in \mathbb{Z}}$ is an element of the $(-\beta)$-shift if and only if for each $n$

$$
\mathrm{d}_{-\beta}\left(-\frac{\beta}{\beta+1}\right) \preceq \text { alt } w_{n} w_{n+1} \cdots \preceq_{\text {alt }} \mathrm{d}_{-\beta}^{*}\left(\frac{1}{\beta+1}\right) .
$$

Put $\mathbf{d}=\mathbf{d}_{-\beta}\left(-\frac{\beta}{\beta+1}\right)=d_{1} d_{2} \cdots$ and $\mathbf{d}^{*}=\mathbf{d}_{-\beta}^{*}\left(\frac{1}{\beta+1}\right)$. Theorem 5.3 shows in particular that $\mathbf{d}$ is an alternately shift minimal word, and the result can be restated as follows.

Lemma 5.4 If $\mathbf{d}=\mathrm{d}_{-\beta}\left(-\frac{\beta}{\beta+1}\right)$ is not a periodic sequence with odd period, then

$$
S_{-\beta}=S(\mathbf{d})=\left\{\left(w_{i}\right)_{i \in \mathbb{Z}} \in A_{\beta}^{\mathbb{Z}} \mid \forall n, \mathbf{d} \preceq_{a l t} w_{n} w_{n+1} \cdots\right\} .
$$

If $\mathbf{d}=\mathbf{d}_{-\beta}\left(-\frac{\beta}{\beta+1}\right)$ is a periodic sequence of odd period, then $\mathbf{d}^{*}=\left(0 d_{1} \cdots d_{2 p}\left(d_{2 p+1}-1\right)\right)^{\omega}$ and

$$
S_{-\beta}=S(\mathbf{d}) \cap S^{\prime}\left(\mathbf{d}^{*}\right)
$$

where

$$
S^{\prime}\left(\mathbf{d}^{*}\right)=\left\{\left(w_{i}\right)_{i \in \mathbb{Z}} \in A_{\beta}^{\mathbb{Z}} \mid \forall n, w_{n} w_{n+1} \cdots \preceq_{\text {alt }} \mathbf{d}^{*}\right\}
$$


Theorem 5.5 The $(-\beta)$-shift is a system of finite type if and only if $\mathrm{d}_{-\beta}\left(-\frac{\beta}{\beta+1}\right)$ is purely periodic.

Proof: If $\mathrm{d}_{-\beta}\left(-\frac{\beta}{\beta+1}\right)$ is purely periodic with an even period, the result follows from Theorem 5.3, Lemma 5.4 and Proposition 4.4. If $\mathrm{d}_{-\beta}\left(-\frac{\beta}{\beta+1}\right)$ is purely periodic with an odd period, the result follows from Theorem 5.3, Lemma 5.4, Proposition 4.4, Remark 4.5, and the fact that the intersection of two finite sets is finite.

By Theorem 5.3, Lemma 5.4, Proposition 4.3, Remark 4.5, and the fact that the intersection of two regular sets is again regular the following result follows.

Theorem 5.6 (Ito-Sadahiro [10]) The $(-\beta)$-shift is a sofic system if and only if $\mathrm{d}_{-\beta}\left(-\frac{\beta}{\beta+1}\right)$ is eventually periodic.

Example 5.7 Let $G=\frac{1+\sqrt{5}}{2}$; then $\mathrm{d}_{G}(1)=11$ and the $G$-shift is offinite type. Since $\mathrm{d}_{-G}\left(-\frac{G}{G+1}\right)=10^{\omega}$ the $(-G)$-shift is a sofic system which is not of finite type.

The automaton in Fig. 4 (right) recognizing the $(-G)$-shift is obtained by minimizing the result of the construction of Proposition 4.2. Remark that it is the automaton that recognizes the celebrated even shift (see [14]).
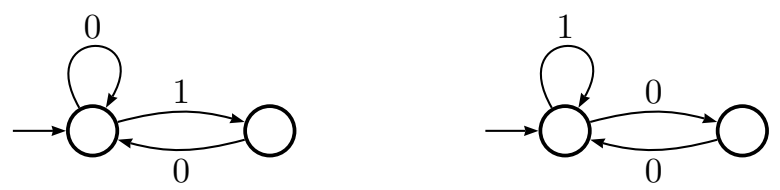

Fig. 4: Finite automata for the $G$-shift (left) and for the $(-G)$-shift (right)

Example 5.8 Let $\beta=G^{2}=\frac{3+\sqrt{5}}{2}$; then $\mathrm{d}_{\beta}(1)=21^{\omega}$ and the $\beta$-shift is sofic, but not of finite type. Now, $\mathrm{d}_{-\beta}\left(-\frac{\beta}{\beta+1}\right)=(21)^{\omega}$ and the $(-\beta)$-shift is of finite type: the set of minimal forbidden factors is $X\left(S_{-\beta}\right)=\{20\}$.
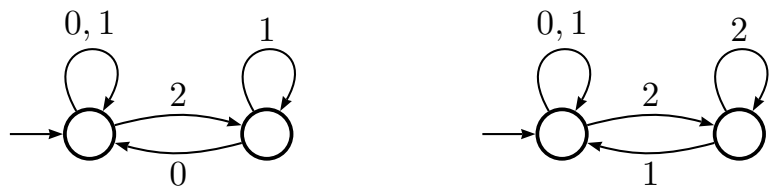

Fig. 5: Finite automata for the $G^{2}$-shift (left) and for the $\left(-G^{2}\right)$-shift (right)

\subsection{Entropy of the $-\beta$-shift}

Examples 5.7 and 5.8 suggest that the entropy of the $(-\beta)$-shift is the same as the entropy of the $\beta$-shift because the adjacency matrices of the automata are the same. This is what we show in this section.

A standard technique for computing the entropy of a subshift $S$ is to construct a (not necessarily finite) automaton recognizing $F(S)$. Then the submatrices of the adjacency matrix are taken into account and for every $n$ the greatest eigenvalue $\lambda_{n}$ of the submatrix of order $n$ is computed. A result proved in [9] ensures that the limit $\lambda$ of the sequence $\lambda_{n}$ exists and it satisfies $h(S)=\log \lambda$. Unfortunately the explicit 
computation of the $\lambda_{n}$ 's in the general case turns out to be very complicated, so we use tools from the theory of dynamical systems:

- the notion of topological entropy for one-dimensional dynamical systems, a one-dimensional dynamical system being a couple $(I, T)$ consisting in a bounded interval $I$ and a piecewise continuous transformation $T: I \rightarrow I$;

- a result by Takahashi [24] establishing the relation between topological entropies of one-dimensional dynamical systems and symbolic dynamical systems;

- a result by Shultz [22] on the topological entropy of some one-dimensional dynamical systems.

Let us begin with the definition of topological entropy for one-dimensional dynamical systems.

Definition 5.9 Let $(I, T)$ be a dynamical system. For every finite cover of $I$, say $\mathcal{C}$, set:

$$
H(T, \mathcal{C}):=\limsup \frac{1}{n} \log N\left(\bigvee_{m=0}^{n-1} T^{-m} \mathcal{C}\right)
$$

with $\bigvee$ denoting the finest common refinement and $N=N(\mathcal{C})$ denoting the number of elements of the smallest subcover of $\mathcal{C}$, a subcover of $\mathcal{C}$ being a subfamily of $\mathcal{C}$ still covering $I$.

The topological entropy of $(I, T)$ is given by the formula

$$
h(I, T):=\sup H(T, \mathcal{C}) .
$$

In [24] Takahashi proved the equality between the topological entropy of a piecewise continuous dynamical system and the topological entropy of an appropriate subshift. Before stating such a result we need a definition.

Definition 5.10 Let $T: I \rightarrow I$ be a piecewise continuous map on the interval $I$. Lap intervals are closed intervals $I_{0}, \ldots, I_{\ell}$ of $T$ satisfying the following conditions:

(a) $I_{0} \cup \cdots \cup I_{\ell}=I$;

(b) $T$ is monotone on each interval $I_{i}, i=0, \ldots, \ell$;

(c) the number $\ell$ is minimal under the conditions $(a)$ and (b).

The number $\ell$ is called lap number and it is denoted lap $(T)$.

Remark 5.11 If the map $T$ is piecewise linear then the lap intervals are unique and they coincide with the intervals of continuity of $T$.

Theorem 5.12 (Takahashi [24]) Let $(I, T)$ be a dynamical system such that $T$ is a piecewise continuous transformation over the closed interval I on itself. Let $A$ be an alphabet and let $\gamma_{T}: I \rightarrow A^{\mathbb{N}}$ be the

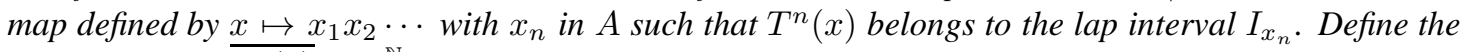
subshift $X_{T}:=\overline{\gamma_{T}(I)}$ in $A^{\mathbb{N}}$.

If lap $(T)$ is finite then

$$
h\left(X_{T}\right)=h(I, T) .
$$


The entropy in the very particular case of a piecewise linear map with constant slope is explicitely given in the following result.

Proposition 5.13 (Shultz [22, Proposition 3.7]) Let $(I, T)$ be a dynamical system such that $T$ is a piecewise linear map with slope $\pm \beta$. Then the topological entropy of $(I, T)$ is equal to $\log \beta$.

We now prove our result.

Theorem 5.14 The topological entropy of $S_{-\beta}$ is equal to $\log \beta$.

Proof: Consider the dynamical system $\left(I_{-\beta}, T_{-\beta}\right)$. We extend the map $T_{-\beta}$ to the closure of $I_{-\beta}$ to fullfill the conditions of Theorem 5.12. By definition of the $(-\beta)$-expansion, the subshift $X_{T_{-}}$coincides with the closure of the set of the $(-\beta)$-expansions in $A_{-\beta}^{\mathbb{N}}$, whose entropy is the same as $S_{-\beta} \subset A_{-\beta}^{\mathbb{Z}}$. As $T_{-\beta}$ is piecewise linear, the lap intervals coincide with the (finite) number of continuity intervals. Then, by Theorem 5.12 and Proposition 5.13, $h\left(S_{-\beta}\right)=h\left(I_{-\beta}, T_{-\beta}\right)=\log \beta$.

\subsection{The Pisot case}

We first prove that the classical result saying that if $\beta$ is a Pisot number, then every element of $\mathbb{Q}(\beta) \cap[0,1]$ has an eventually periodic $\beta$-expansion is still valid for the base $-\beta$.

Theorem 5.15 If $\beta$ is a Pisot number, then every element of $\mathbb{Q}(\beta) \cap I_{-\beta}$ has an eventually periodic $(-\beta)$-expansion.

Proof: Let $M_{\beta}(X)=X^{d}-a_{1} X^{d-1}-\cdots-a_{d}$ be the minimal polynomial of $\beta$ and denote by $\beta=$ $\beta_{1}, \ldots, \beta_{d}$ the conjugates of $\beta$. Let $x$ be arbitrarily fixed in $\mathbb{Q}(\beta) \cap I_{-\beta}$. Since $\mathbb{Q}(\beta)=\mathbb{Q}(-\beta), x$ can be expressed as $x=q^{-1} \sum_{i=0}^{d-1} p_{i}(-\beta)^{i}$ with $q$ and $p_{i}$ in $\mathbb{Z}, q>0$ as small as possible in order to have uniqueness.

Let $\left(x_{i}\right)_{i \geqslant 1}$ be the $(-\beta)$-expansion of $x$, and write

$$
r_{n}=r_{n}^{(1)}=r_{n}^{(1)}(x)=\frac{x_{n+1}}{-\beta}+\frac{x_{n+2}}{(-\beta)^{2}}+\cdots=(-\beta)^{n}\left(x-\sum_{k=1}^{n} x_{k}(-\beta)^{-k}\right) .
$$

Since $r_{n}=T_{-\beta}^{n}(x)$ belongs to $I_{-\beta}$ then $\left|r_{n}\right| \leqslant \frac{\beta}{\beta+1}<1$. For $2 \leqslant j \leqslant d$, let

$$
r_{n}^{(j)}=r_{n}^{(j)}(x)=\left(-\beta_{j}\right)^{n}\left(q^{-1} \sum_{i=0}^{d-1} p_{i}\left(-\beta_{j}\right)^{i}-\sum_{k=1}^{n} x_{k}\left(-\beta_{j}\right)^{-k}\right) .
$$

Let $\eta=\max \left\{\left|\beta_{j}\right| \mid 2 \leqslant j \leqslant d\right\}$ : since $\beta$ is a Pisot number, $\eta<1$. Since $x_{k} \leqslant\lfloor\beta\rfloor$ we get

$$
\left|r_{n}^{(j)}\right| \leqslant q^{-1} \sum_{i=0}^{d-1}\left|p_{i}\right| \eta^{n+i}+\lfloor\beta\rfloor \sum_{k=0}^{n-1} \eta^{k}
$$

and since $\eta<1, \max _{1 \leqslant j \leqslant d}\left\{\sup _{n}\left\{\left|r_{n}^{(j)}\right|\right\}\right\}<\infty$.

We need a technical result. Set $R_{n}=\left(r_{n}^{(1)}, \ldots, r_{n}^{(d)}\right)$ and let $B$ the matrix $B=\left(\left(-\beta_{j}\right)^{-i}\right)_{1 \leqslant i, j \leqslant d}$. 
Lemma 5.16 Let $x=q^{-1} \sum_{i=0}^{d-1} p_{i}(-\beta)^{i}$. For every $n \geqslant 0$ there exists a unique $d$-uple $Z_{n}=\left(z_{n}^{(1)}, \ldots, z_{n}^{(d)}\right)$ in $\mathbb{Z}^{d}$ such that $R_{n}=q^{-1} Z_{n} B$.

Proof: By induction on $n$. First, $r_{1}=-\beta x-x_{1}$, thus

$$
r_{1}=q^{-1}\left(\sum_{i=0}^{d-1} p_{i}(-\beta)^{i+1}-q x_{1}\right)=q^{-1}\left(\frac{z_{1}^{(1)}}{-\beta}+\cdots+\frac{z_{1}^{(d)}}{(-\beta)^{d}}\right)
$$

using the fact that $(-\beta)^{d}=-a_{1}(-\beta)^{d-1}+a_{2}(-\beta)^{d-2}+\cdots+(-1)^{d} a_{d}$. Now, $r_{n+1}=-\beta r_{n}-x_{n+1}$, hence

$$
r_{n+1}=q^{-1}\left(z_{n}^{(1)}+\frac{z_{n}^{(2)}}{-\beta}+\cdots+\frac{z_{n}^{(d)}}{(-\beta)^{d-1}}-q x_{n+1}\right)=q^{-1}\left(\frac{z_{n+1}^{(1)}}{-\beta}+\cdots+\frac{z_{n+1}^{(d)}}{(-\beta)^{d}}\right)
$$

since $z_{n}^{(1)}-q x_{n+1} \in \mathbb{Z}$. Thus for every $n$ there exists $\left(z_{n}^{(1)}, \ldots, z_{n}^{(d)}\right)$ in $\mathbb{Z}^{d}$ such that

$$
r_{n}=q^{-1} \sum_{k=1}^{d} z_{n}^{(k)}(-\beta)^{-k}
$$

Since the latter equation has integral coefficients and is satisfied by $-\beta$, it is also satisfied by $-\beta_{j}, 2 \leqslant$ $j \leqslant d$, and

$$
r_{n}^{(j)}=\left(-\beta_{j}\right)^{n}\left(q^{-1} \sum_{i=0}^{d-1} p_{i}\left(-\beta_{j}\right)^{i}-\sum_{k=1}^{n} x_{k}\left(-\beta_{j}\right)^{-k}\right)=q^{-1} \sum_{k=1}^{d} z_{n}^{(k)}\left(-\beta_{j}\right)^{-k} .
$$

Let us go back to the proof of Theorem 5.15. Let $V_{n}=q R_{n}$. The $\left(V_{n}\right)_{n \geqslant 1}$ have bounded norm, since $\max _{1 \leqslant j \leqslant d}\left\{\sup _{n}\left\{\left|r_{n}^{(j)}\right|\right\}\right\}<\infty$. As the matrix $B$ is invertible, for every $n \geqslant 1$,

$$
\left\|Z_{n}\right\|=\left\|\left(z_{n}^{(1)}, \ldots, z_{n}^{(d)}\right)\right\|=\max \left\{\left|z_{n}^{(j)}\right|: 1 \leqslant j \leqslant d\right\}<\infty
$$

so there exist $p$ and $m \geqslant 1$ such that $Z_{m+p}=Z_{p}$, hence $r_{m+p}=r_{p}$ and the $(-\beta)$-expansion of $x$ is eventually periodic.

As a corollary we get the following result.

Theorem 5.17 If $\beta$ is a Pisot number then the (- $\beta)$-shift is a sofic system.

The normalization in base $-\beta$ is the function which maps any $(-\beta)$-representation over an alphabet $C$ of digits of a given number of $I_{-\beta}$ onto the admissible $(-\beta)$-expansion of that number.

Let $C=\{-c, \ldots, c\}$, where $c \geqslant\lfloor\beta\rfloor$ is an integer. Denote

$$
Z_{-\beta}(2 c)=\left\{\left(z_{i}\right)_{i \geqslant 0} \in\{-2 c, \ldots, 2 c\}^{\mathbb{N}} \mid \sum_{i \geqslant 0} z_{i}(-\beta)^{-i}=0\right\}
$$


The set $Z_{-\beta}(2 c)$ is recognized by a countable infinite automaton $\mathcal{A}_{-\beta}(2 c)$ : the set of states $Q(2 c)$ consists of all $s \in \mathbb{Z}[\beta] \cap\left[-\frac{2 c}{\beta-1}, \frac{2 c}{\beta-1}\right]$. Transitions are of the form $s \stackrel{e}{\rightarrow} s^{\prime}$ with $e \in\{-c, \ldots, c\}$ such that $s^{\prime}=-\beta s+e$. The state 0 is initial; every state is terminal.

Let $M_{\beta}(X)$ be the minimal polynomial of $\beta$, and denote by $\beta=\beta_{1}, \beta_{2}, \ldots, \beta_{d}$ the roots of $M_{\beta}$. We define a norm on the discrete lattice of rank $d, \mathbb{Z}[X] /\left(M_{\beta}\right)$, as

$$
\|P(X)\|=\max _{1 \leqslant i \leqslant d}\left|P\left(\beta_{i}\right)\right| .
$$

Proposition 5.18 If $\beta$ is a Pisot number then the automaton $\mathcal{A}_{-\beta}(2 c)$ is finite for every $c \geqslant\lfloor\beta\rfloor$.

Proof: Every state $s$ in $Q(2 c)$ is associated with the label of the shortest path $f_{0} f_{1} \cdots f_{n}$ from 0 to $s$ in the automaton. Thus $s=f_{0}(-\beta)^{n}+f_{1}(-\beta)^{n-1}+\cdots+f_{n}=P(\beta)$, with $P(X)$ in $\mathbb{Z}[X] /\left(M_{\beta}\right)$. Since $f_{0} f_{1} \cdots f_{n}$ is a prefix of a word of $Z_{-\beta}(2 c)$, there exists $f_{n+1} f_{n+2} \cdots$ such that $\left(f_{i}\right)_{i \geqslant 0}$ is in $Z_{-\beta}(2 c)$. Thus $s=|P(\beta)|<\frac{2 c}{\beta-1}$. For every conjugate $\beta_{i}, 2 \leqslant i \leqslant d,\left|\beta_{i}\right|<1$, and $\left|P\left(\beta_{i}\right)\right|<\frac{2 c}{1-\mid \beta_{i}}$. Thus every state of $Q(2 c)$ is bounded in norm, and so there is only a finite number of them.

The redundancy transducer $\mathcal{R}_{-\beta}(c)$ is similar to $\mathcal{A}_{-\beta}(2 c)$. Each transition $s \stackrel{e}{\rightarrow} s^{\prime}$ of $\mathcal{A}_{-\beta}(2 c)$ is replaced in $\mathcal{R}_{-\beta}(c)$ by a set of transitions $s \stackrel{a \mid b}{\longrightarrow} s^{\prime}$, with $a, b \in\{-c, \ldots, c\}$ and $a-b=e$. Thus one obtains the following proposition.

Proposition 5.19 The redundancy transducer $\mathcal{R}_{-\beta}(c)$ recognizes the set

$$
\left\{\left(x_{1} x_{2} \cdots, y_{1} y_{2} \cdots\right) \in C^{\mathbb{N}} \times C^{\mathbb{N}} \mid \sum_{i \geqslant 1} x_{i}(-\beta)^{-i}=\sum_{i \geqslant 1} y_{i}(-\beta)^{-i}\right\} .
$$

If $\beta$ is a Pisot number, then $\mathcal{R}_{-\beta}(c)$ is finite.

Theorem 5.20 If $\beta$ is a Pisot number, then normalization in base $-\beta$ on any alphabet $C$ is realizable by a finite transducer.

Proof: The normalization is obtained by keeping in $\mathcal{R}_{-\beta}(c)$ only the outputs $y$ that are $(-\beta)$-admissible. By Theorem 5.17 the set of admissible words is recognizable by a finite automaton $\mathcal{D}_{-\beta}$. The finite transducer $\mathcal{N}_{-\beta}(c)$ doing the normalization is obtained by making the intersection of the output automaton of $\mathcal{R}_{-\beta}(c)$ with $\mathcal{D}_{-\beta}$.

Proposition 5.21 If $\beta$ is a Pisot number, then the conversion from base $-\beta$ to base $\beta$ is realizable by a finite transducer. The result is $\beta$-admissible.

Proof: Let $x \in I_{-\beta}, x \geqslant 0$, such that $\mathrm{d}_{-\beta}(x)=x_{1} x_{2} x_{3} \cdots$. Denote $\bar{a}$ the signed digit $(-a)$. Then $\overline{x_{1}} x_{2} \overline{x_{3}} \ldots$ is a $\beta$-representation of $x$ on the alphabet $\overline{A_{-\beta}}=\{-\lfloor\beta\rfloor, \ldots,\lfloor\beta\rfloor\}$. Thus the conversion is equivalent to the normalization in base $\beta$ on the alphabet $\widetilde{A_{-\beta}}$, and when $\beta$ is a Pisot number, it is realizable by a finite transducer by [4]. 


\section{On-line conversion from positive to negative base}

Proposition 5.21 shows the actability of the conversion from negative to positive base with a finite transducer for a particular class of bases, i.e., the Pisot numbers. The result is admissible, but this transducer is not sequential.

In the case where the base is a negative integer, we have seen in Section 3 that the conversion from base $b$ to base $-b$ is realizable by a finite right sequential transducer.

\subsection{On-line conversion in the general case}

An on-line algorithm is such that, after a certain delay of latency $\delta$ during which the data are read without writing, a digit of the output is produced for each digit of the input, see [17] for on-line arithmetic in integer base.

Theorem 6.1 There exists a conversion from base $\beta$ to base $-\beta$ which is computable by an on-line algorithm with delay $\delta$, where $\delta$ is the smallest positive integer such that

$$
\frac{\lfloor\beta\rfloor}{\beta^{\delta-1}}+\frac{\lfloor\beta\rfloor}{\beta^{\delta}} \leqslant 1-\{\beta\}
$$

In general the result is not $(-\beta)$-admissible.

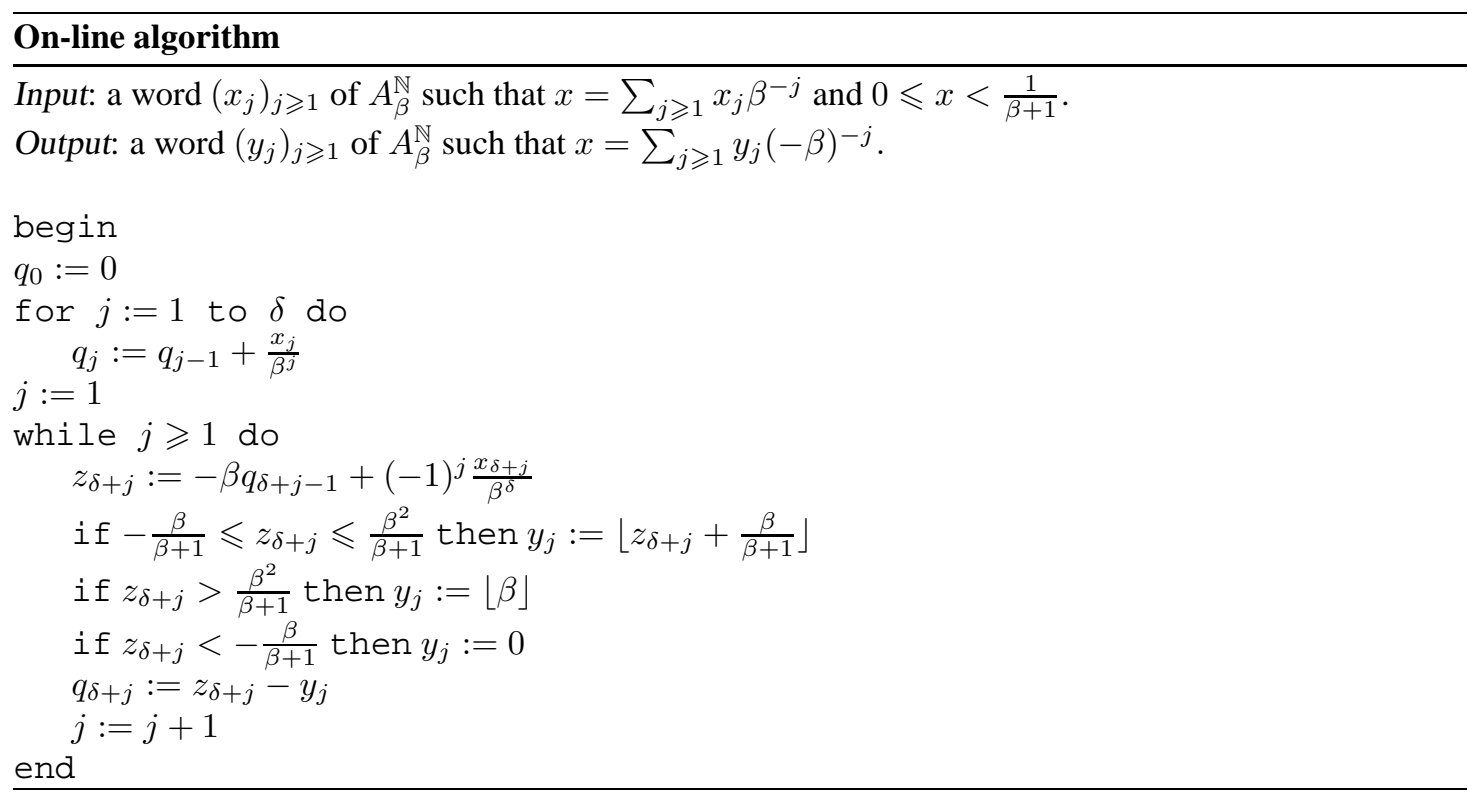

Proof: Claim 1. For each $j \geqslant 1$

$$
\frac{x_{1}}{\beta}+\frac{x_{2}}{\beta^{2}}+\cdots+\frac{x_{\delta+j}}{\beta^{\delta+j}}=-\frac{y_{1}}{\beta}+\frac{y_{2}}{\beta^{2}}-\cdots+(-1)^{j} \frac{y_{j}}{\beta^{j}}+(-1)^{j} \frac{q_{\delta+j}}{\beta^{j}} .
$$

Claim 2. If $-\frac{\beta}{\beta+1} \leqslant z_{\delta+j} \leqslant \frac{\beta^{2}}{\beta+1}$ then $y_{j}$ belongs to $A_{\beta}$ and $q_{\delta+j}$ belongs to $I_{-\beta}=\left[-\frac{\beta}{\beta+1}, \frac{1}{\beta+1}\right)$. Proof of Claim 2: Clearly $0 \leqslant y_{j} \leqslant \frac{\beta^{2}}{\beta+1}+\frac{\beta}{\beta+1}=\beta$. Moreover, $z_{\delta+j}+\frac{\beta}{\beta+1}=y_{j}+\left\{z_{\delta+j}+\frac{\beta}{\beta+1}\right\}$, thus 
$q_{\delta+j}:=z_{\delta+j}-y_{j}=\left\{z_{\delta+j}+\frac{\beta}{\beta+1}\right\}-\frac{\beta}{\beta+1}$, and the claim is proved.

Claim 3. If $z_{\delta+j}>\frac{\beta^{2}}{\beta+1}$ then $q_{\delta+j}>-\frac{\beta}{\beta+1}$.

Proof of Claim 3: We have that $q_{\delta+j}=z_{\delta+j}-\lfloor\beta\rfloor>\frac{\beta^{2}}{\beta+1}-\lfloor\beta\rfloor>-\frac{\beta}{\beta+1}$.

Claim 4. If $z_{\delta+j}>\frac{\beta^{2}}{\beta+1}$ and $q_{\delta+j-1} \geqslant-\frac{\beta}{\beta+1}$ then $q_{\delta+j}<\frac{1}{\beta+1}$.

Proof of Claim 4: Since $q_{\delta+j}=-\beta q_{\delta+j-1}+(-1)^{j} \frac{x_{\delta+j}}{\beta^{\delta}}-\lfloor\beta\rfloor \leqslant \frac{\beta^{2}}{\beta+1}+\frac{\lfloor\beta\rfloor}{\beta^{\delta}}-\lfloor\beta\rfloor$, the claim is proved if, and only if, $\frac{\lfloor\beta\rfloor}{\beta^{\delta}}-\lfloor\beta\rfloor<1-\beta$, that is to say, if, and only if, $\frac{\lfloor\beta\rfloor}{\beta^{\delta}}<1-\{\beta\}$, which is true thanks to (3).

Claim 5. If $z_{\delta+j}<-\frac{\beta}{\beta+1}$ and $q_{\delta+j-1} \in I_{-\beta}$ then $j$ is odd, $-\frac{\beta}{\beta+1}-\frac{\lfloor\beta\rfloor}{\beta^{\delta}} \leqslant q_{\delta+j}<-\frac{\beta}{\beta+1}$, and $q_{\delta+j+1}$ belongs to $I_{-\beta}$.

Proof of Claim 5: If $j$ is even then $z_{\delta+j}:=-\beta q_{\delta+j-1}+\frac{x_{\delta+j}}{\beta^{\delta}}>-\frac{\beta}{\beta+1}+\frac{x_{\delta+j}}{\beta^{\delta}} \geqslant-\frac{\beta}{\beta+1}$, hence $j$ must be odd. Set $j=2 k+1$. We have $y_{2 k+1}=0$ and $q_{\delta+2 k+1}=z_{\delta+2 k+1}=-\beta q_{\delta+2 k}-\frac{x_{\delta+2 k+1}}{\beta^{\delta}} \geqslant-\frac{\beta}{\beta+1}-\frac{\lfloor\beta\rfloor}{\beta^{\delta}}$ since $q_{\delta+j-1} \in I_{-\beta}$.

Then $z_{\delta+2 k+2}=-\beta q_{\delta+2 k+1}+\frac{x_{\delta+2 k+2}}{\beta^{\delta}}>\frac{\beta^{2}}{\beta+1}$. Hence $y_{2 k+2}=\lfloor\beta\rfloor$. By Claim 3, $q_{\delta+2 k+2}>-\frac{\beta}{\beta+1}$. On the other hand $q_{\delta+2 k+2}=z_{\delta+2 k+2}-\lfloor\beta\rfloor=-\beta q_{\delta+2 k+1}+\frac{x_{\delta+2 k+2}}{\beta^{\delta}}-\lfloor\beta\rfloor=\beta^{2} q_{\delta+2 k}+\frac{x_{\delta+2 k+1}}{\beta^{\delta-1}}+$ $\frac{x_{\delta+2 k+2}}{\beta^{\delta}}-\lfloor\beta\rfloor<\frac{\beta^{2}}{\beta+1}+\frac{\lfloor\beta\rfloor}{\beta^{\delta-1}}+\frac{\lfloor\beta\rfloor}{\beta^{\delta}}-\lfloor\beta\rfloor \leqslant \frac{1}{\beta+1}$ by (3), thus $q_{\delta+2 k+2}$ belongs to $I_{-\beta}$.

By hypothesis, $q_{\delta}$ is in $I_{-\beta}$. By the previous claims, for every $k \geqslant 0, q_{\delta+2 k}$ belongs to $I_{-\beta}$ and $-\frac{\beta}{\beta+1}-\frac{\lfloor\beta\rfloor}{\beta^{\delta}} \leqslant q_{\delta+2 k+1}<\frac{1}{\beta+1}$. Thus, for every $j \geqslant 1$,

$$
\frac{x_{1}}{\beta}+\cdots+\frac{x_{\delta+j}}{\beta^{\delta+j}}=\frac{y_{1}}{(-\beta)}+\cdots+\frac{y_{j}}{(-\beta)^{j}}+\frac{q_{\delta+j}}{(-\beta)^{j}}
$$

with $q_{\delta+j}$ bounded. Therefore the algorithm converges, and

$$
\sum_{j \geqslant 1} x_{j} \beta^{-j}=\sum_{j \geqslant 1} y_{j}(-\beta)^{-j}
$$

\subsection{Conversion in the Pisot case}

We now show that, when $\beta$ is a Pisot number, there is a finite on-line transducer realizing the conversion.

Theorem 6.2 If $\beta$ is a Pisot number, the conversion from base $\beta$ to base $-\beta$ is realizable by a finite on-line transducer.

Proof: Following the on-line algorithm of Section 6.1 we construct an on-line transducer $\mathcal{C}$ as follows. The set of states is $Q=Q_{t} \cup Q_{s}$, with the set of transient states $Q_{t}=\left\{q_{j} \mid 0 \leqslant j \leqslant \delta-1\right\}$, and the set of synchronous states $Q_{s}=\left\{q_{\delta+j} \mid j \geqslant 0\right\}$. The initial state is $q_{0}$. For $1 \leqslant j \leqslant \delta$, transient edges are defined by

$$
q_{j-1} \stackrel{x_{j} \mid \varepsilon}{\longrightarrow} q_{j}
$$


Synchronous edges are defined by

$$
q_{\delta+j-1} \stackrel{x_{\delta+j} \mid y_{j}}{\longrightarrow} q_{\delta+j}
$$

for $j \geqslant 1$. There is an infinite path in the automaton $\mathcal{C}$ starting in $q_{0}$ and labelled by

$$
q_{0} \stackrel{x_{1} \mid \varepsilon}{\longrightarrow} q_{1} \cdots \stackrel{x_{\delta} \mid \varepsilon}{\longrightarrow} q_{\delta} \stackrel{x_{\delta+1} \mid y_{1}}{\longrightarrow} q_{\delta+1} \stackrel{x_{\delta+2} \mid y_{2}}{\longrightarrow} q_{\delta+2} \cdots
$$

if, and only if, $\sum_{j \geqslant 1} x_{j} \beta^{-j}=\sum_{j \geqslant 1} y_{j}(-\beta)^{-j}$.

Let $M_{\beta}(X)$ be the minimal polynomial of $\beta$ and let $\beta=\beta_{1}, \beta_{2}, \ldots, \beta_{d}$ be the roots of $M_{\beta}$. Recall that $\mathbb{Z}[X] /\left(M_{\beta}(X)\right) \sim \mathbb{Z}[\beta]$ is a discrete lattice of rank $d$. Since $\beta$ is a Pisot number, $\left|\beta_{i}\right|<1$ for $2 \leqslant i \leqslant d$.

For each $j \geqslant 1, q_{j}$ is an element of $\mathbb{Z}\left[\beta, \beta^{-1}\right]$. For $1 \leqslant i \leqslant d$ let $q_{j}\left(\beta_{i}\right)$ be the element of $\mathbb{Z}\left[\beta_{i}, \beta_{i}^{-1}\right]$ obtained by replacing $\beta$ by $\beta_{i}$ in $q_{j}$. Then $q_{j}=q_{j}(\beta)$.

First of all, for every $j \geqslant 1,-\frac{\beta}{\beta+1}-\frac{\lfloor\beta\rfloor}{\beta^{\delta}} \leqslant q_{j}(\beta)<\frac{1}{\beta+1}$ by the on-line algorithm.

Secondly, for every $j \geqslant 1$ and $2 \leqslant i \leqslant d$,

$$
q_{\delta+j}\left(\beta_{i}\right)=-\beta_{i} q_{\delta+j-1}\left(\beta_{i}\right)+(-1)^{j} \frac{x_{\delta+j}}{\beta_{i}^{\delta}}-y_{j} .
$$

For $2 \leqslant i \leqslant d$ let

$$
M_{i}=\frac{\lfloor\beta\rfloor}{\left(1-\left|\beta_{i}\right|\right)}\left(1+\frac{1}{\left|\beta_{i}\right|^{\delta}}\right) .
$$

Then, if $\left|q_{\delta+j-1}\left(\beta_{i}\right)\right| \leqslant M_{i}$, then $\left|q_{\delta+j}\left(\beta_{i}\right)\right| \leqslant M_{i}$ by (4).

Now, for $0 \leqslant j \leqslant \delta$ and $2 \leqslant i \leqslant d$,

$$
\left|q_{j}\left(\beta_{i}\right)\right|<\lfloor\beta\rfloor\left(\frac{1}{\left|\beta_{i}\right|}+\cdots+\frac{1}{\left|\beta_{i}\right|^{\delta}}\right)<M_{i}
$$

Define a norm on $\mathbb{Z}[X] /\left(M_{\beta}(X)\right)$ by $\|q\|=\max _{1 \leqslant i \leqslant d}\left|q\left(\beta_{i}\right)\right|$. Thus the elements of $Q$ are all bounded in norm, and so $Q$ is finite.

In the particular case that $\beta^{2}=a \beta+1, a$ integer $\geqslant 1$ ( $\beta$ is thus a Pisot number), we can construct directly a simpler finite left sequential transducer realizing the conversion.

Proposition 6.3 If $\beta^{2}=a \beta+1, a \geqslant 1$, then the conversion from base $\beta$ to base $-\beta$ is realizable by the finite left sequential transducer of Fig. 6 .

Proof: The left sequential transducer in Fig. 6 converts a $\beta$-expansion of a real number $x$ in $[0, \beta)$ of the form $x_{0} \cdot x_{1} x_{2} \cdots$ into a $(-\beta)$-representation of $x$ of the form $y_{0} \cdot y_{1} y_{2} \cdots$. The processing is done from left to right by 2 -letter blocks. We take $0 \leqslant d \leqslant a, 0 \leqslant c \leqslant a-1,1 \leqslant e \leqslant a$. Since the input is admissible, no factor $a e$, with $1 \leqslant e \leqslant a$ can occur in an input word. 


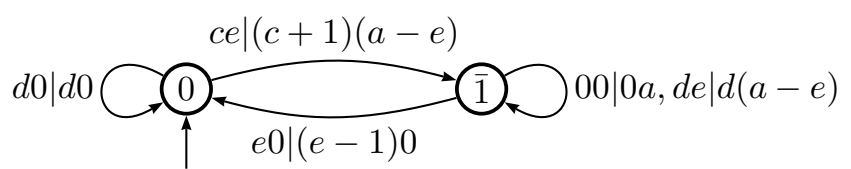

Fig. 6: Finite left sequential transducer realizing conversion from base $\beta$ to base $-\beta, \beta^{2}=a \beta+1$

\section{References}

[1] P. Ambrož, D. Dombek, Z. Masáková, E. Pelantová, Numbers with integer expansion in the numeration system with negative base, preprint 2009, 13pp. http: / / arxiv.org/abs / 0912.4597.

[2] A. Bertrand, Développements en base de Pisot et répartition modulo 1, C. R. Acad. Sci. Paris Sér. $A-B 285$ (1977) A419-A421.

[3] S. Eilenberg, Automata, Languages and Machines, vol. A, Academic Press, 1974.

[4] Ch. Frougny, Representations of numbers and finite automata, Math. Systems Theory 25 (1992) 3760.

[5] Ch. Frougny, On-line finite automata for addition in some numeration systems, Theoretical Informatics and Applications 33 (1999) 79-101.

[6] Ch. Frougny and A. C. Lai, On negative bases, in Proceedings of DLT 09, Lectures Notes in Computer Science 5583 (2009) 252-263.

[7] Ch. Frougny and J. Sakarovitch, Number representation and finite automata, Chapter 2 in Combinatorics, Automata and Number Theory, V. Berthé, M. Rigo (Eds), Encyclopedia of Mathematics and its Applications 135, Cambridge University Press, 2010.

[8] V. Grünwald, Intorno all' aritmetica dei sistemi numerici a base negativa con particolare riguardo al sistema numerico a base negativo-decimale per lo studio delle sue analogie coll' aritmetica ordinaria (decimale), Giornale di Matematiche di Battaglini 367 (1885) 203-221.

[9] F. Hofbauer, Maximal measures for piecewise monotonically increasing transformations on [0,1], in Ergodic Theory, eds. M. Denker and K. Jacobs, Lecture Notes in Mathematics 729, Springer, Berlin, 66-77 (1979).

[10] S. Ito and T. Sadahiro, (- $\beta$ )-expansions of real numbers, INTEGERS 9 (2009) 239-259.

[11] S. Ito and Y. Takahashi, Markov subshifts and realization of $\beta$-expansions, J. Math. Soc. Japan 26 (1974) 33-55.

[12] D.E. Knuth, The Art of Computer Programming, Seminumerical Algorithms, Vol.2, 2nd ed. AddisonWesley (1988).

[13] L. Liao and W Steiner, Dynamical properties of the negative beta transformation, preprint (2011). 
[14] D. Lind and B. Marcus, An introduction to symbolic dynamics and coding, Cambridge University Press, 1995.

[15] M. Lothaire, Algebraic combinatorics on words, Encyclopedia of Mathematics and its Applications 90, Cambridge University Press, Cambridge, 2002.

[16] Z. Masáková, E. Pelantová, T. Vavrá, Arithmetics in number systems with negative base, Theor. Comp. Sci. (2011), to appear, http://arxiv.org/abs/1002.1009.

[17] J.-M.Muller, Some characterizations of functions computable in on-line arithmetic. I.E.E.E. Trans. on Computers 43 (1994) 752-755.

[18] W. Parry, On the $\beta$-expansions of real numbers, Acta Math. Acad. Sci. Hungar. 11 (1960) 401-416.

[19] A. Rényi, Representations for real numbers and their ergodic properties, Acta Math. Acad. Sci. Hungar. 8 (1957) 477-493.

[20] J. Sakarovitch, Elements of Automata Theory, Cambridge University Press, 2009.

[21] K. Schmidt, On periodic expansions of Pisot numbers and Salem numbers, Bull. London Math. Soc. 12 (1980) 269-278.

[22] F. Shultz, Dimension groups for interval maps. II. The transitive case Ergodic Theory Dynam. Systems 27 (2007) 1287-1321.

[23] W. Steiner, On the structure of $(-\beta)$-integers, preprint 2010, $15 \mathrm{pp}$. http: //arxiv.org/abs/1011.1755.

[24] Y. Takahashi, A formula for topological entropy of one-dimensional dynamics Sci. Papers College Gen. Ed. Univ. Tokyo 30 (1980) 11-22. 
